\title{
Lifestyle Factors, Unspecified
}

National Cancer Institute

\section{Source}

National Cancer Institute. Lifestyle Factors, Unspecified. NCI Thesaurus. Code C20201.

The consideration of unspecified lifestyle factors as variables in disease incident, transmission, and control. 